\title{
A New Piezoelectric Actuator Induces Bone Formation In Vivo: A Preliminary Study
}

\author{
Joana Reis, ${ }^{1}$ Clara Frias, ${ }^{2}$ Carlos Canto e Castro, ${ }^{3}$ \\ Maria Luísa Botelho, ${ }^{3}$ António Torres Marques, ${ }^{2}$ José António Oliveira Simões, ${ }^{4}$ \\ Fernando Capela e Silva, ${ }^{5,6}$ and José Potes ${ }^{1,6}$ \\ ${ }^{1}$ Departamento de Medicina Veterinária, Universidade de Évora, 7002-554 Évora, Portugal \\ ${ }^{2}$ Departamento de Engenharia Mecânica, Faculdade de Engenharia, Universidade do Porto, Rua Roberto Frias, \\ 4200-465 Porto, Portugal \\ ${ }^{3}$ Instituto Tecnológico e Nuclear, Estrada Nacional 10, 2686-953 Sacavém, Portugal \\ ${ }^{4}$ Departamento de Mecânica, Universidade de Aveiro, Campus Universitário de Santiago, 3810-193 Aveiro, Portugal \\ ${ }^{5}$ Departamento de Biologia, Universidade de Évora, 7002-554 Évora, Portugal \\ ${ }^{6}$ Instituto de Ciências Agrárias e Ambientais Mediterrânicas (ICAAM), Universidade de Évora, 7002-554 Évora, Portugal
}

Correspondence should be addressed to Joana Reis, jmfcr@uevora.pt

Received 11 January 2012; Revised 21 March 2012; Accepted 9 April 2012

Academic Editor: Chuanyue Wu

Copyright ( $\odot 2012$ Joana Reis et al. This is an open access article distributed under the Creative Commons Attribution License, which permits unrestricted use, distribution, and reproduction in any medium, provided the original work is properly cited.

\begin{abstract}
This in vivo study presents the preliminary results of the use of a novel piezoelectric actuator for orthopedic application. The innovative use of the converse piezoelectric effect to mechanically stimulate bone was achieved with polyvinylidene fluoride actuators implanted in osteotomy cuts in sheep femur and tibia. The biological response around the osteotomies was assessed through histology and histomorphometry in nondecalcified sections and histochemistry and immunohistochemistry in decalcified sections, namely, through Masson's trichrome, and labeling of osteopontin, proliferating cell nuclear antigen, and tartrate-resistant acid phosphatase. After one-month implantation, total bone area and new bone area were significantly higher around actuators when compared to static controls. Bone deposition rate was also significantly higher in the mechanically stimulated areas. In these areas, osteopontin increased expression was observed. The present in vivo study suggests that piezoelectric materials and the converse piezoelectric effect may be used to effectively stimulate bone growth.
\end{abstract}

\section{Introduction}

It is known that bone mass density and architecture are intimately dependent upon mechanical factors. Several in vitro studies suggest that cell mechanical stimulation promotes proliferation and differentiation of osteogenic lineage [1,2], in agreement with the results from several in vivo studies [3-5]. Bone remodeling is influenced by strain magnitude, frequency, and loading duration.

The clinical longevity and success of an implant also depend on mechanical determinants. Stress shielding causes bone loss through remodeling due to decreased loading, particularly in noncemented prostheses $[6,7]$. Several solutions have been proposed for these two mechanical problems, namely, design variations and development of controlled-stiffness implants $[8,9]$. A new approach to this problem, with potential application to other orthopedic problems and other medical fields, would be the development of smart implants integrating systems for bone mechanical stimulation.

The present in vivo study explores the use of the converse piezoelectric effect to mechanically stimulate bone with resource to Polyvinylidene Fluoride films (PVDF) (Measurement Specialties Inc Company, USA). These films were used, after dip-coating to improve cell adhesion and assure electrical insulation, as a substrate for MC3T3 cells to explore the concept of using piezoelectric materials and converse piezoelectric effect to produce effective osteoblast mechanical stimulation. The coating was performed by dip-coating at constant velocity of $0,238 \mathrm{~mm} / \mathrm{s}$. The chosen material for coating was an acrylic, poly(methyl methacrylate) (PMMA), (PERFEX, International Dental Products, USA), used alone 


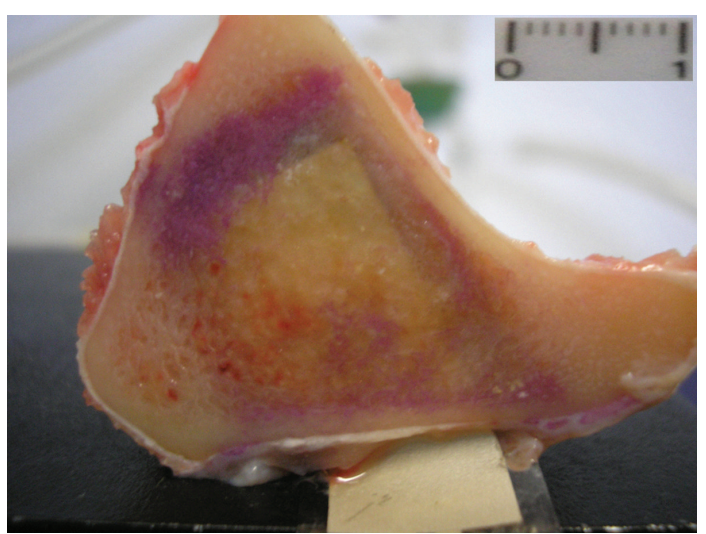

(a)

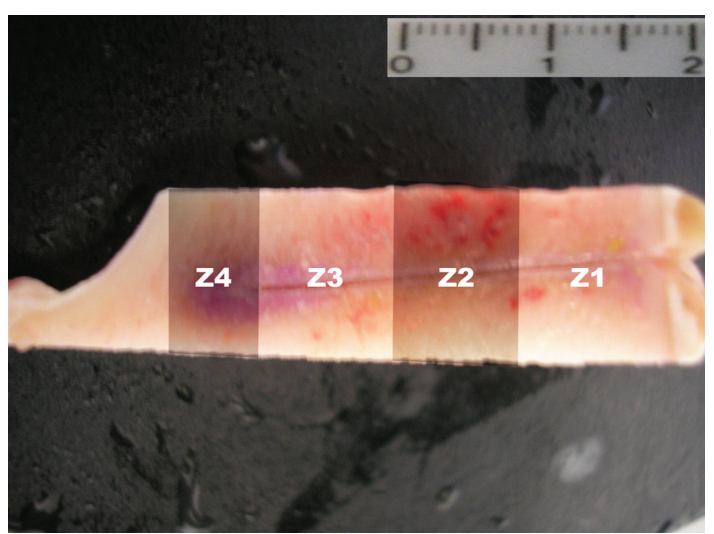

(b)

FIgURE 1: Location of the actuators within the bone. The figure shows example of bone section prior to inclusion: (a) section of tibia-scale bar $1 \mathrm{~cm}$; (b) shows how the areas for histomorphometry were distributed; $\mathrm{Z} 1$ corresponds to the film clamped region—scale bar $2 \mathrm{~cm}$.

in the first three layers and a in forth layer along with $4 \%$ of Bonelike $(250-500 \mu \mathrm{m})$ particles added (kindly offered by INESCPorto). The displacement was higher where the cells were seeded, in the central area of the coated devices, in the order of $700 \mathrm{~nm}$ along the $z$-axis, in a semisinusoidal fashion. On the substrates submitted to dynamic conditions, stimulation was done with an alternating sinusoidal current (AC), of $5 \mathrm{~V}$, at $1 \mathrm{~Hz}$ and $3 \mathrm{~Hz}$ for 15 minutes at each frequency [10]. In this exploratory in vivo study, the same coating procedures and daily stimulation regimens were applied. The biological response around the osteotomies was assessed through histology and histomorphometry in undecalcified sections, and through histochemistry and immunohistochemistry in decalcified sections, namely, through Masson's trichrome, and labeling of osteopontin (OPN), proliferating cell nuclear antigen (PCNA), and tartrate-resistant acid phosphatase (TRAP).

\section{Materials and Methods}

2.1. Actuator Device Design and Sterilization. The actuator device was composed of a microboard containing an ultralow power 16-bit microcontroller (eZ430-RF2500, Texas Instruments, USA), powered by lithium battery and encapsulated in PMMA and a set of six actuators composed of polyvinylidene fluoride (PVDF) and silver electrodes, electrically insulated by dip-coating as described elsewhere [10]. Each actuator measured $15 \mathrm{~mm}$ width and $40 \mathrm{~mm}$ length, with an active area of $12 \times 31 \mathrm{~mm}^{2}$. The actuator device was programmed to apply a $5 \mathrm{~V}$ square wave of $1 \mathrm{~Hz}$ and $3 \mathrm{~Hz}$ for 15 minutes at each frequency, once every 24 hours.

Finite Numerical Method studies and Electronic Speckle Pattern Interferometry Process (ESPI) studies have shown the lower deformation levels occurred in the clamped region of the films, for a potential differential of $5 \mathrm{~V}$, and the higher deformation occurred in the opposite extremity of the film [10].

A control device, similar but static was also developed. A sterilization method was developed and validated based on
ISO 11737-1 and ISO 14937, using a series of decontaminating solutions (10\% sodium hypochlorite for 20 minutes, without agitation, washing in sterile water, and immersion in 10\% hydrogen peroxide for 30 minutes, without agitation, followed by rinse in sterile water and drying under laminar flux), before 2 hours of exposure to UV (in two different positions).

2.2. Surgical Procedure. The actuator device was implanted in the left hind limb, and a control device was implanted in the right hind limb, of a 4-year-old merino ewe, with $45 \mathrm{~kg}$ body-weight.

The animal was premedicated with atropine $(0.7 \mathrm{mg} / \mathrm{kg})$, xylazine $(0.1 \mathrm{mg} / \mathrm{kg})$ and butorphanol $(0.01 \mathrm{mg} / \mathrm{kg})$, and anesthesia induced with thiopental sodium $5 \%(5 \mathrm{mg} / \mathrm{kg})$ by intravenous injection. The anesthesia was maintained through isoflurane with spontaneous ventilation.

The stifle joint of both legs was prepared with a solution of povidone-iodine and ethanol 70\%, after being shorn and shaved. Two longitudinal incisions were made on the craniomedial border of the femur and at tibial crest level. The tibial bone was exposed by reflecting deep medial fascia, the vastus medialis and cranial part of the sartorius muscles and the periosteum, and two osteotomies were made using an especially metal designed guide to make two regular and well-orientated osteotomies using an oscillating saw. The bone was continuously irrigated with a sterile saline solution during the process of low-speed drilling and cutting. The same procedure was followed with a different design guide for the distal femoral physis, where four osteotomies were done. The osteotomies were made perpendicularly to the long axis of the bone.

A stab wound was made in the fascia lata, and the extremities of the piezoelectric films passed through it, and then, the active area of each piezoelectric film was placed inside the slot osteotomy cavity. The actuator position inside the tibial bone can be clearly viewed in Figure 1(a), obtained post-mortem. The portion of the device containing the microprocessor and the power supply was left in the nearby subcutaneous space. 


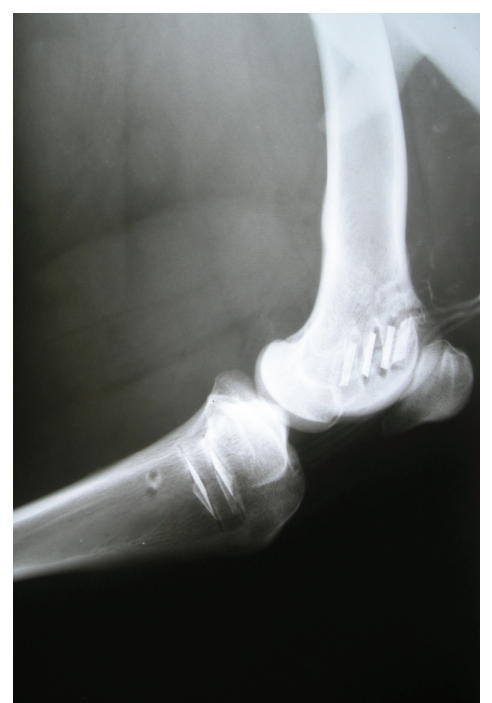

Figure 2: Postoperative radiograph thirty days after implantation showing the six actuators in place (four in the femur, two in the tibia). There are neither signs of periostal or peri-implantar reaction nor signs of infection in neighboring soft tissues.

The actuator device was implanted in the left hind limb, and control on the right hind limb. After placing the implants, incisions were closed routinely with a continuouspattern suture in the retinaculum and fascia, using absorbable Surgicryl 2/0 (SMI AG, Belgium) and in the skin with Silk 0 (SMI AG).

Postoperative X-ray images were obtained thirty days after implantation, before sacrifice, to ensure the proper evolution of the bone cuts and to detect signs of infection/inflammation (Figure 2).

One week after implantation calcein $(15 \mathrm{mg} / \mathrm{kg}$; Sigma, USA) was injected subcutaneously and one week prior to sacrifice the same procedure was done with alizarin complexone $(25 \mathrm{mg} / \mathrm{kg}$; Sigma, USA). Thirty days after implantation, the ewe was sacrificed by intravenous sodium pentobarbital injection.

The present study was authorized by competent national authorities and conducted accordingly to FELASA's guidelines for animal care.

\subsection{Tissue Processing, Histology, and Immunohistochemistry.} Both hind limbs were dissected, the implanted materials and surrounding tissue removed and fixed in $4 \%$ paraformaldehyde for two weeks. Bone samples were cut transversally to the long axis of the bone, each including a piezoelectric film and the surrounding bone (Figure 1(a)).

Specimens were dehydrated through an ascending ethanol series. Soft tissues (local lymph nodes and samples of the fibrous capsule surrounding the implants) were routinely processed and embedded in paraffin. Undecalcified bone samples of each of the implants were included in resin (Technovit 9100, Heraeus Kulzer, Germany) according to the manufacturer's instructions and $80 \mu \mathrm{m}$ thick sections cut with a saw microtome (Leica 1600, Germany) parallel to the piezoelectric film long axis. A minimum of five sections of each resin block was cut. Sections were then appropriately processed for routine staining (Giemsa Eosin), mounted for fluorescence microscopy. The prepared slides were evaluated qualitatively. For histomorphometric studies, the interface between the bone and implant was divided in four distinct areas: Z1, Z2, Z3, and Z4, from cortical towards bone surrounding the free extremity of the piezoelectric film (Figure 1(b)). Pictures were taken from the bone surrounding both sides of the film in areas $\mathrm{Z} 1$ to $\mathrm{Z} 3$ and Z4, using a 40x magnification (Figure 3).

For immunohistochemistry and histochemistry, bone sections were decalcified in formic acid 5\% for 3 weeks, dehydrated in ethanol, cleared in xylene, and embedded in paraffin wax and $3 \mu \mathrm{m}$ sections cut. After deparaffinization and rehydration, immunohistochemistry sections were treated with 3\% hydrogen peroxide for 10 minutes. Primary antibodies for Tartrate-Resistant Acid Phosphatase (TRAP) (Thermo Scientific, USA, mouse monoclonal antibody, Ab1, Clone 26E5, Cat. no.MS-1768), Proliferating Cell Nuclear Antigen (PCNA) (NeoMarkers, USA, mouse monoclonal antibody, Ab-1, Clone PC10, Cat. no.MS-106-P0), and Osteopontin (NeoMarkers, rabbit polyclonal antibody, Cat. no.RB-9097) were diluted to $1: 20,1: 200$, and $1: 50$, respectively. Prior to immunostaining, the sections were pretreated for antigen retrieval at $100^{\circ} \mathrm{C}$ in $10 \mathrm{mM}$ citrate buffer, $\mathrm{pH} 6$, for 20 minutes in microwave oven, followed by cooling for 30 minutes at room temperature. For double staining of PCNA and osteopontin, immunohistochemistry was done using a double staining kit PicTure (Zymed Laboratories Inc, USA), according with the manufacturer's instructions. Slides were counterstained with Mayer's hematoxylin and Clearmount used to mount the slides. TRAP immunohystochemistry was performed with resource to kit PicTure-MAX Polymer (Invitrogen, USA). Slides were counterstained with Mayer's hematoxilin, dehydrated, mounted with Entellan (Merck, Germany), and coverslipped.

For all sections, positive controls were made simultaneously. As negative controls adjacent sections were incubated: (a) without primary antibody and (b) with rabbit/rat normal serum (similar concentration as that of primary).

Masson Trichrome was done using a kit (Bio-Optica, Italy), followed by dehydration and mounting.

Observation and pictures were done using optic (Olympus BX41) and fluorescence microscope equipped with WB Fluorescence cube (Olympus U-LH100HG).

2.4. Statistical Analysis. Total bone area, immature bone area, and mature bone area were measured using Sigma ScanPro 5 (Systat Software Inc., USA). The results, in percentage of total bone area, mature bone, and immature bone, were determined for each piezoelectric film in the predetermined areas Z1 to Z4. Capsule thickness was measured with resource to cell ${ }^{\wedge} \mathrm{A}$ software (Olympus, Germany). Normal distribution of the results was verified using the Kolmogorov-Smirnov test, homogeneity of variance assessed through the Levene test, and differences between groups tested using one-way ANOVA (at a 0.05 level). The statistical analysis was done using software OriginPro 8 (OriginLab Corporation, USA). 

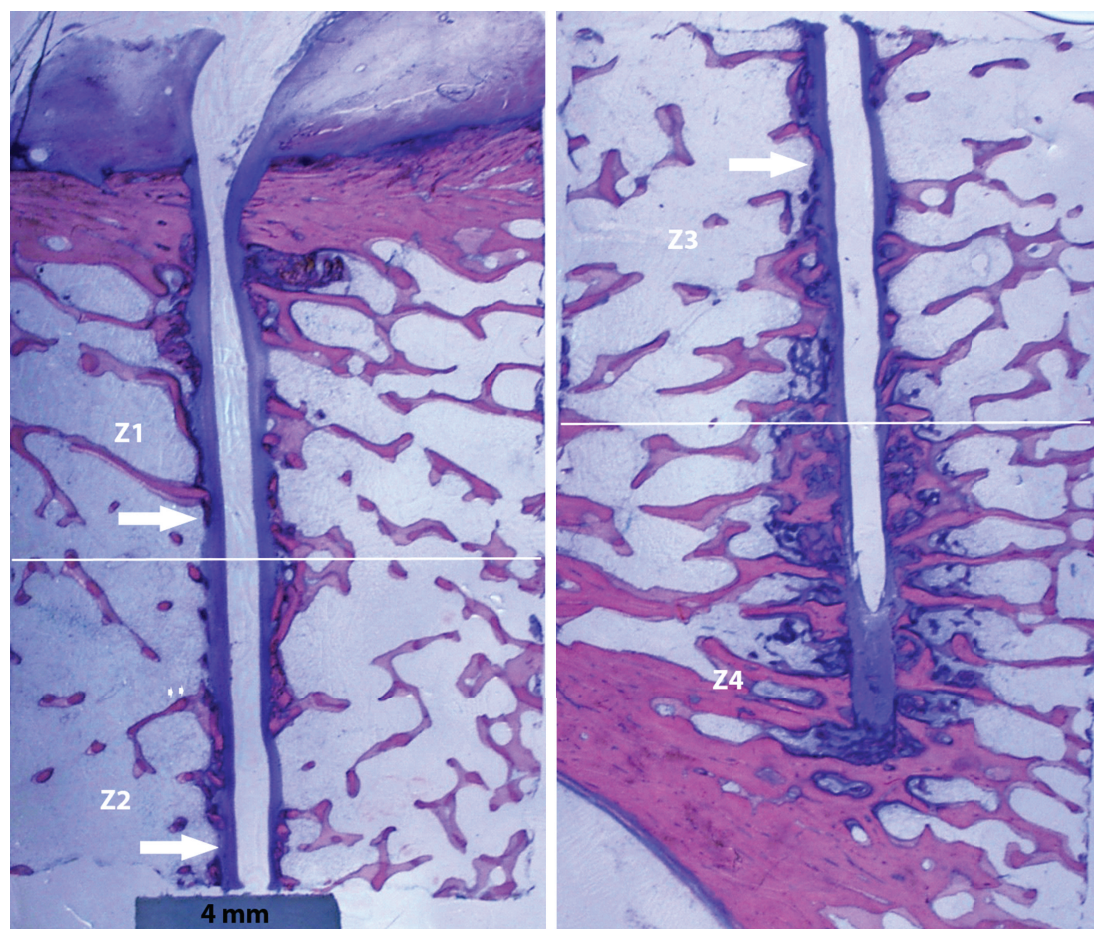

FIGURE 3: Low-magnification picture of histological section where it is evident the fibrous tissue around the implant (arrows). Scale bar represents $4 \mathrm{~mm}$.

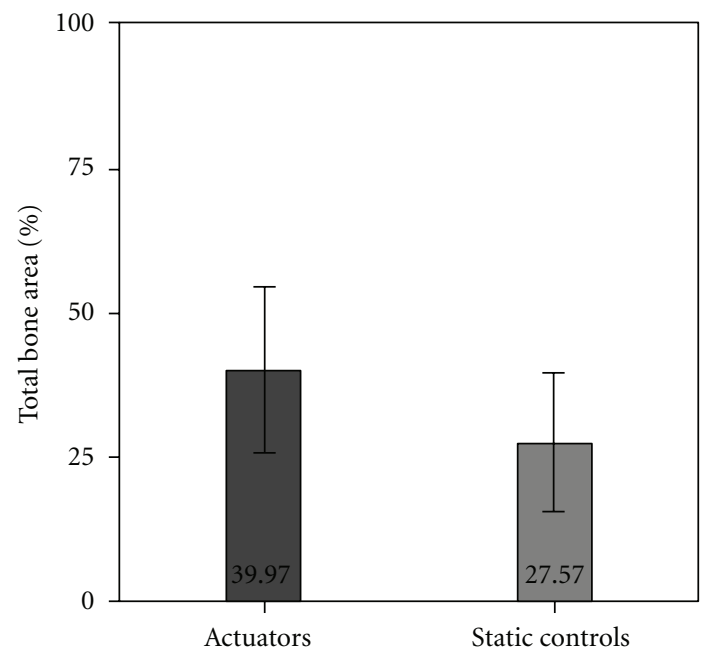

FIgURE 4: Total bone area measured around actuators and static controls, expressed in percentage. Bars represent means and error bars standard deviation.

\section{Results}

After one-month implantation period, there were statistically significant differences. Total bone area around the actuators was significantly higher, when comparing to static controls $(39.91 \pm 14.08 \%$ versus $27.20 \pm 11.98 \%)$ (Figure 4$)$.

The increment of bone occupied area was due to new bone formation, and in actuators the area occupied by woven bone and osteoid was $64.89 \pm 19.32 \%$ of the total bone area versus $31.72 \pm 14.54 \%$ in static devices (Figure 5 ). The chosen staining technique, Giemsa Eosin, stains older bone in a darker shade of pink, whilst more recently deposed bone is of a lighter pink color and frequently adjacent to much less prominent osteoid (dark blue) areas (Figures 5(a) and 5(b)). Taking into account the close relation in between osteoid and woven, recently formed bone, the authors chose not to make separated measurements that could be misleading. The bone marrow present in the sections showed no relevant alterations.

The deposition of new bone was more marked in certain areas of the devices. The significant differences arise from the bone areas surrounding the two distal thirds of the devices; around the devices' clamped area, no differences were found between actuated and static devices (Figure 6).

When assessing the mineral apposition rate in the distal third of the films, with the aid of the fluorochrome labeling, the mineral apposition rate was significantly higher around actuated devices $(4.44 \pm 1.67 \mu \mathrm{m} /$ day $)$ than around static devices $(2.70 \pm 0.95 \mu \mathrm{m} /$ day) (Figure 5).

A fibrous capsule was obvious both in actuators and static devices, with no statistical significant differences in capsule thickness between the two groups $(289.59 \pm 131.20 \mu \mathrm{m}$ in actuated films versus $293.93 \pm 84.79 \mu \mathrm{m})$. The average thickness of the capsule was of $292 \mu \mathrm{m}$.

Immunohistochemistry failed to detect differences in TRAP expression between the two groups, but there is a marked elevation in OPN detection around actuators, in $\mathrm{Z3}$ and Z4 (Figure 7). OPN is also strongly labeled in the fibroblast layers closer to the PVDF films, both in actuators and static controls, and this is the area where positive PCNA labeling was found more often. PCNA labeling was also 


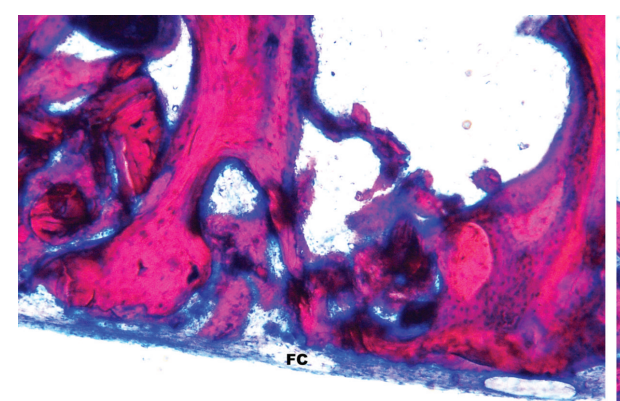

a)

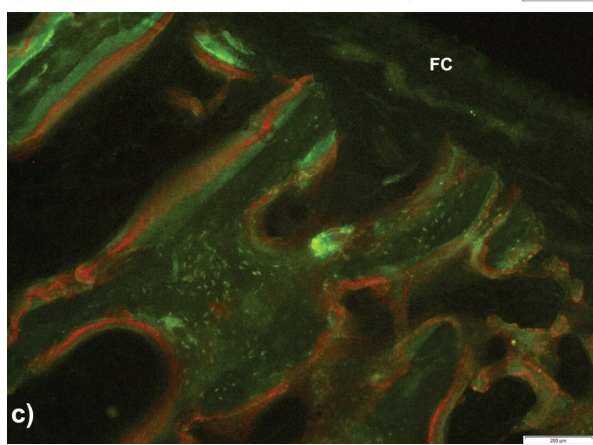

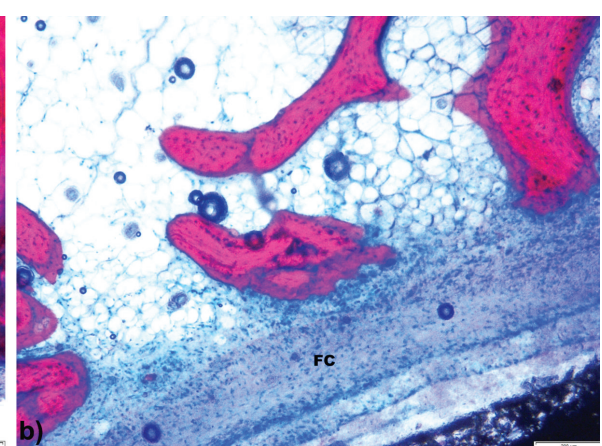

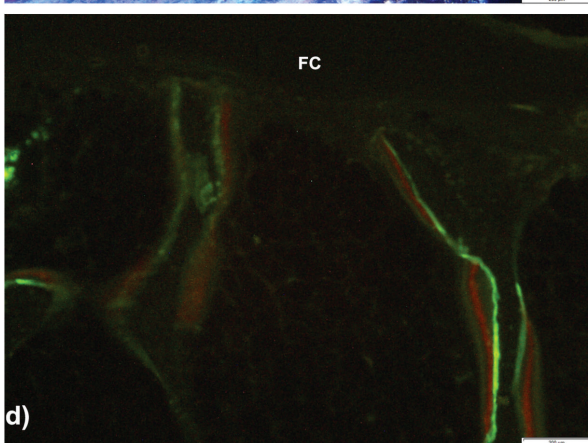

FIGURE 5: Microphotograph of nondecalcified sections. On top, (a) shows Z3 areas of actuator and (b) shows static control, both implanted in the same position in tibia. On bottom, Z2 areas showing increase labeled area in (c) femoral actuator when compared to contra lateral (d) static control. A fibrous capsule (FC) was present on the bone/film interface. Scale bar represents $200 \mu \mathrm{m}$.

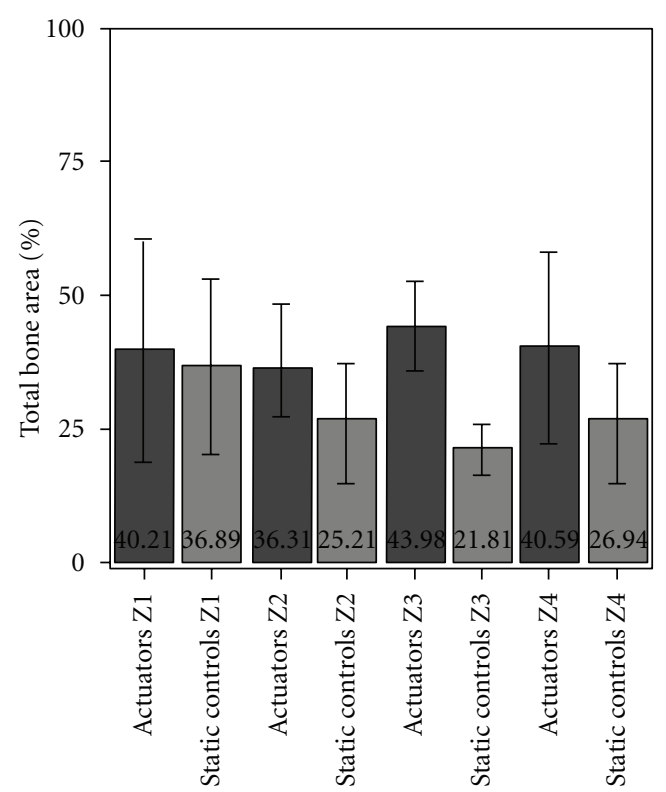

FIGURE 6: Total bone area measured around actuators and static controls, organized by areas Z1 to Z4. Bars represent means and error bars standard deviation.

positive in some of the preosteoblastic cells in layers apposed to OPN-positive columnar osteoblasts.

\section{Discussion}

The existence of micro-movements at the interface, depending on the magnitude, may enhance osteogenesis or impair it and promote the formation of fibrous tissue $[11,12]$. Considering the deformation levels of the actuator devices, under the experimental conditions, the generated strains were low, but the results suggest they are enough to elicit response.

Mineral apposition rate and total bone mass are significantly higher around actuated films when compared to static controls, and these differences arise from around the areas of the films known to have higher displacement values.

The increased OPN expression we found around actuators when comparing to static controls is most likely associated with mechanical stress, and it was not accompanied by a proportional increase in TRAP. OPN is a multifunctional noncollagenous glycoprotein present in bone matrix, expressed in various degrees by proliferating preosteoblasts, osteoblasts, and osteocytes but also by fibroblasts, osteoclasts, and macrophages [13-15]. OPN production is known to be increased in association with mechanical loading [15, 16], and its deficiency significantly decreases bone fracture toughness, leading to heterogeneous mineral distribution, since OPN is known to bind strongly to hydroxyapatite, and cell surface as well $[17,18]$.

However, a few questions arise. The first is related to PVDF's biocompatibility. We observed that all the devices were separated from neighboring bone by a fibrous capsule. This is probably due to the material itself, since there were no differences between actuators and static controls. It would be mandatory to develop and test a material with improved biocompatibility to evaluate accurately the bone material interface.

One other issue arises from reflecting upon the material and the coating properties and behavior. The in vitro study 

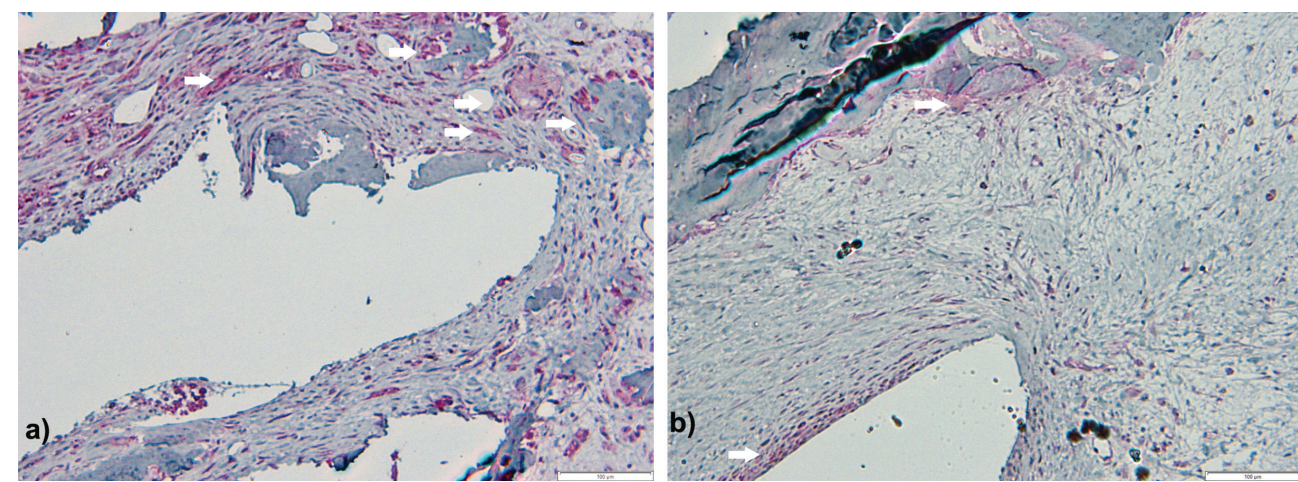

FIgURE 7: Microphotograph of decalcified sections, osteopontin (white arrows) and PCNA expression. Picture shows Z3 areas of femoral: (a) actuator and (b) static control, suggesting more extensive osteopontin labeling around actuator. Double Fast-Red and DAB immunohistochemistry staining for osteopontin and PCNA, respectively. Scale bar represents $100 \mu \mathrm{m}$.

previously developed shows evidence of adequate electric insulation for 48 hours in the experimental conditions [10].

However, the in vivo environment is not as controlled and, in addition to a longer period of time, the implantation procedure itself may induce damage on the coating. If coating damage occurs, one should consider the possible effects of the material (PVDF and silver nitrate particles from the electrode) and electrical current. Previous studies have shown cytotoxic silver nitrate activity, dependent on the concentration $[19,20]$.

The possible creation of electric field locally and its effects are more complex. The clinical advantages of applying electrical and electromagnetic fields in the treatment of fractures and mal-unions have been long known although the mechanisms leading to the clinical response are not well understood [21-23]. Electric fields as low as $0.1 \mathrm{mV} / \mathrm{cm}$ promote osteoblast proliferation, with cell metabolic activity being influenced by the intensity of the electric field, and cell signaling based on the voltage-dependent calcium channels $[24,25]$. Electric and electromagnetic fields also exert effects on fibroblasts, enhancing proliferation, protein synthesis and influencing cell alignment, by calcium-independent mechanisms [26-29]. The fact that we found no significant differences in fibrous capsule thickness surrounding the devices suggests it is likely that the electric insulation remained satisfactory, but further studies would be needed to prove this. However, if an electric field was being created locally, increased bone formation along the whole actuator could also be expected. Instead, new bone formation follows a pattern in accordance with what is known to be the areas of increased motion and deformation.

This study was performed in one animal only but using an adequate number of devices (total of twelve). The sheep was chosen as an animal model considering the bone biology similarities with the human species and the size of the devices. The results are very clear in evidencing qualitative and quantitative statistically significant differences when comparing static and actuated films, but it would be necessary to enlarge the animal study and to study response with longer implantation times. However, from an ethical point of view, it would be desirable to do so after developing a material other than PVDF, more biocompatible.

\section{Conclusions}

In conclusion, the present in vivo study suggests that in fact piezoelectric materials and the converse piezoelectric effect may be used to effectively stimulate bone growth at the bone implant interface, eliciting an early response. Piezoelectric materials have the advantages of enabling a wide range of deformation/displacement, frequencies, and regimens. Therefore, they are excellent candidate components of actuators for clinical purposes. However, new materials should be developed, aiming better biocompatibility.

\section{Conflict of Interests}

The authors have no potential conflict of interests. This paper has not been submitted or published elsewhere, although its content is included in a Ph.D. thesis entitled "The bone/implant interface: new approaches to old problems," submitted in the University of Évora, Portugal.

\section{Funding}

The Portuguese Science Foundation (FCT) supports this paper (projects PTDC/EME-PME/65749/2006, PTDC/EMEPME/70824/2006, and Grant BD/31895/ 2006). However, FCT played no role in the development of the present work or upon its submission for publication.

\section{Authors' Contribution}

J. Reis and C. Frias contributed equally in the development of this paper.

\section{Acknowledgments}

This paper is funded by FEDER Funds through the Operational Programme for Competitiveness Factors-COMPETE and National Funds through FCT-Foundation for Science and Technology under the Strategic Project PEst-C/AGR/ UI0115/2011. Authors acknowledge also the financial support from the Portuguese Science Foundation (FCT) in 
the forms of projects PTDC/EME-PME/65749/2006 and PTDC/EME-PME/70824/2006 and Ph.D. Grant BD/31895/ 2006 of Joana Reis. The Portuguese Science Foundation (FCT) played no role in the development of the present work or upon its submission for publication.

\section{References}

[1] M. Lewandowska-Szumieł, K. Sikorski, A. Szummer, Z. Lewandowski, and W. Marczyński, "Osteoblast response to the elastic strain of metallic support," Journal of Biomechanics, vol. 40, no. 3, pp. 554-560, 2007.

[2] A. Kadow-Romacker, J. E. Hoffmann, G. Duda, B. Wildemann, and G. Schmidmaier, "Effect of mechanical stimulation on osteoblast- and osteoclast-like cells in vitro," Cells Tissues Organs, vol. 190, no. 2, pp. 61-68, 2009.

[3] C. T. Rubin and L. E. Lanyon, "Regulation of bone formation by applied dynamic loads," Journal of Bone and Joint Surgery A, vol. 66, no. 3, pp. 397-402, 1984.

[4] S. Bourrin, S. Palle, R. Pupier, L. Vico, and C. Alexandre, "Effects of physical training on bone adaptation in three zones of the rat tibia," Journal of Bone and Mineral Research, vol. 10, no. 11, pp. 1745-1752, 1995.

[5] Y.-F. Hsieh and C. H. Turner, "Effects of loading frequency on mechanically induced bone formation," Journal of Bone and Mineral Research, vol. 16, no. 5, pp. 918-924, 2001.

[6] R. Huiskes, H. Weinans, and B. Rietbergen, "The relationship between stress shielding and bone resorption around total hip stems and the effects of flexible materials," Clinical Orthopaedics and Related Research, no. 274, pp. 124-134, 1992.

[7] L. I. Havelin, B. Espehaug, S. E. Vollset, and L. B. Engesaeter, "Early failures among 14,009 cemented and 1,326 uncemented prostheses for primary coxarthrosis: the Norwegian Arthroplasty Register, 1987-1992," Acta Orthopaedica Scandinavica, vol. 65, no. 1, pp. 1-6, 1994.

[8] J. A. Simões, A. T. Marques, and G. Jeronimidis, "Design of a controlled-stiffness composite proximal femoral prosthesis," Composites Science and Technology, vol. 60, no. 4, pp. 559-567, 2000.

[9] M. Campbell, M. N. Bureau, and L. H. Yahia, "Performance of CF/PA12 composite femoral stems," Journal of Materials Science, vol. 19, no. 2, pp. 683-693, 2008.

[10] C. Frias, J. Reis, F. Capela e Silva, J. Potes, J. Simões, and A. T. Marques, "Polymeric piezoelectric actuator substrate for osteoblast mechanical stimulation," Journal of Biomechanics, vol. 43, no. 6, pp. 1061-1066, 2010.

[11] R. Huiskes, W. D. V. Driel, P. J. Prendergast, and K. Søballe, "A biomechanical regulatory model for periprosthetic fibroustissue differentiation," Journal of Materials Science, vol. 8, no. 12, pp. 785-788, 1997

[12] P. Leucht, J. B. Kim, R. Wazen et al., "Effect of mechanical stimuli on skeletal regeneration around implants," Bone, vol. 40, no. 4, pp. 919-930, 2007.

[13] G. J. Pinero, M. C. Farach-Carson, R. E. Devoll, J. E. Aubin, J. C. Brunn, and W. T. Butler, "Bone matrix proteins in osteogenesis and remodelling in the neonatal rat mandible as studied by immunolocalization of osteopontin, bone sialoprotein, alpha2 HS-glycoprotein and alkaline phosphatase," Archives of Oral Biology, vol. 40, no. 2, pp. 145-155, 1995.

[14] N. Ashizawa, K. Graf, Y. S. Do et al., "Osteopontin is produced by rat cardiac fibroblasts and mediates A(II)- induced DNA synthesis and collagen gel contraction," Journal of Clinical Investigation, vol. 98, no. 10, pp. 2218-2227, 1996.
[15] D. S. Perrien, E. C. Brown, J. Aronson et al., "Immunohistochemical study of osteopontin expression during distraction osteogenesis in the rat," Journal of Histochemistry and Cytochemistry, vol. 50, no. 4, pp. 567-574, 2002.

[16] L. V. Harter, K. A. Hruska, and R. L. Duncan, "Human osteoblast-like cells respond to mechanical strain with increased bone matrix protein production independent of hormonal regulation," Endocrinology, vol. 136, no. 2, pp. 528-535, 1995.

[17] L. W. Fisher, D. A. Torchia, B. Fohr, M. F. Young, and N. S. Fedarko, "Flexible structures of SIBLING proteins, bone sialoprotein, and osteopontin," Biochemical and Biophysical Research Communications, vol. 280, no. 2, pp. 460-465, 2001.

[18] P. J. Thurner, C. G. Chen, S. Ionova-Martin et al., "Osteopontin deficiency increases bone fragility but preserves bone mass," Bone, vol. 46, no. 6, pp. 1564-1573, 2010.

[19] E. Hidalgo and C. Domínguez, "Study of cytotoxicity mechanisms of silver nitrate in human dermal fibroblasts," Toxicology Letters, vol. 98, no. 3, pp. 169-179, 1998.

[20] V. K. M. Poon and A. Burd, "In vitro cytotoxity of silver: implication for clinical wound care," Burns, vol. 30, no. 2, pp. 140-147, 2004.

[21] J. T. Ryaby, "Clinical effects of electromagnetic and electric fields on fracture healing," Clinical Orthopaedics and Related Research, no. 355, pp. S205-S215, 1998.

[22] J. B. Haddad, A. G. Obolensky, and P. Shinnick, "The biologic effects and the therapeutic mechanism of action of electric and electromagnetic field stimulation on bone and cartilage: new findings and a review of earlier work," Journal of Alternative and Complementary Medicine, vol. 13, no. 5, pp. 485-490, 2007.

[23] C. Goldstein, S. Sprague, and B. A. Petrisor, "Electrical stimulation for fracture healing: current evidence," Journal of Orthopaedic Trauma, vol. 24, no. 1, pp. S62-S65, 2010.

[24] C. T. Brighton, E. Okereke, S. R. Pollack, and C. C. Clark, "In vitro bone-cell response to a capacitively coupled electrical field: the role of field strength, pulse pattern, and duty cycle," Clinical Orthopaedics and Related Research, no. 285, pp. 255262, 1992.

[25] C. T. Brighton, W. Wang, R. Seldes, G. Zhang, and S. R. Pollack, "Signal transduction in electrically stimulated bone cells," Journal of Bone and Joint Surgery A, vol. 83, no. 10, pp. 1514-1523, 2001.

[26] M. J. Brown and L. M. Loew, "Electric field-directed fibroblast locomotion involves cell surface molecular reorganization and is calcium independent," Journal of Cell Biology, vol. 127, no. 1, pp. 117-128, 1994.

[27] C. A. Erickson and R. Nuccitelli, "Embryonic fibroblast motility and orientation can be influenced by physiological electric fields," Journal of Cell Biology, vol. 98, no. 1, pp. 296-307, 1984.

[28] G. J. Bourguignon and L. Y. W. Bourguignon, "Electric stimulation of protein and DNA synthesis in human fibroblasts," The FASEB Journal, vol. 1, no. 5, pp. 398-402, 1987.

[29] E. Finkelstein, W. Chang, P. H. G. Chao et al., "Roles of microtubules, cell polarity and adhesion in electric-field-mediated motility of 3T3 fibroblasts," Journal of Cell Science, vol. 117, no. 8, pp. 1533-1545, 2004. 


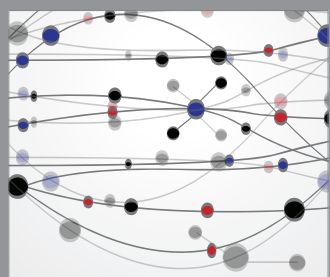

The Scientific World Journal
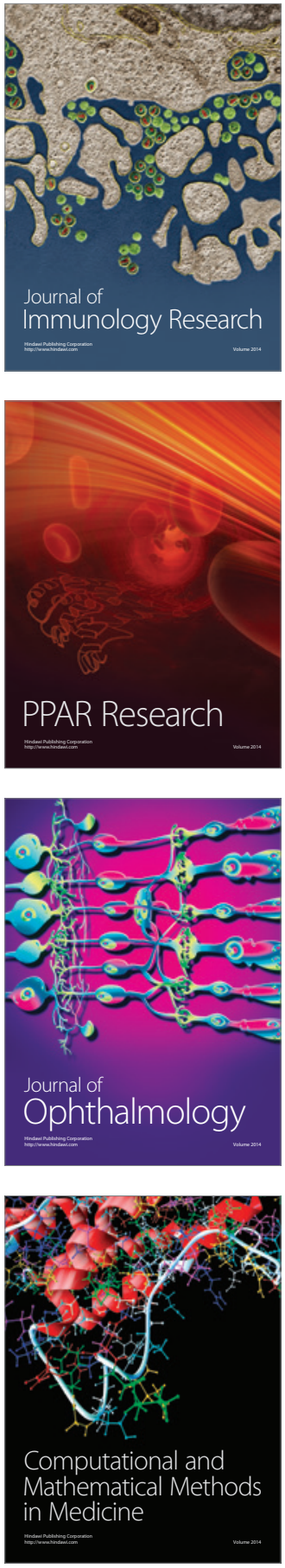

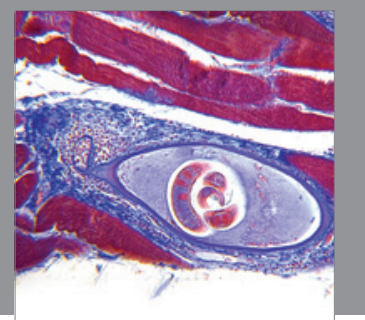

Gastroenterology

Research and Practice
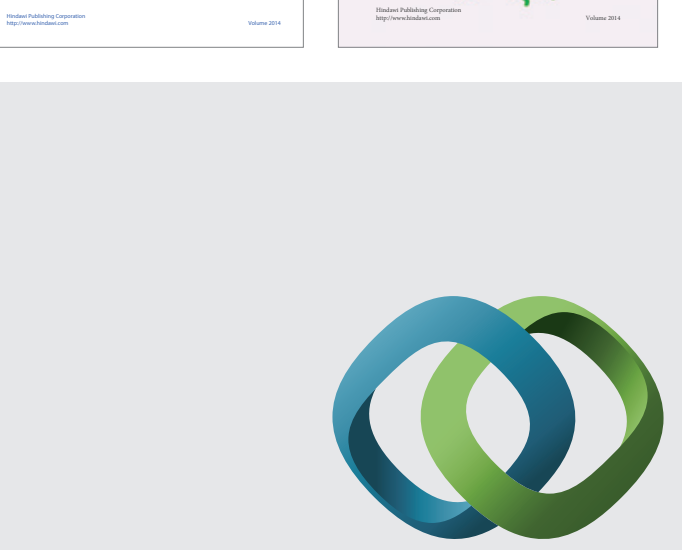

\section{Hindawi}

Submit your manuscripts at

http://www.hindawi.com
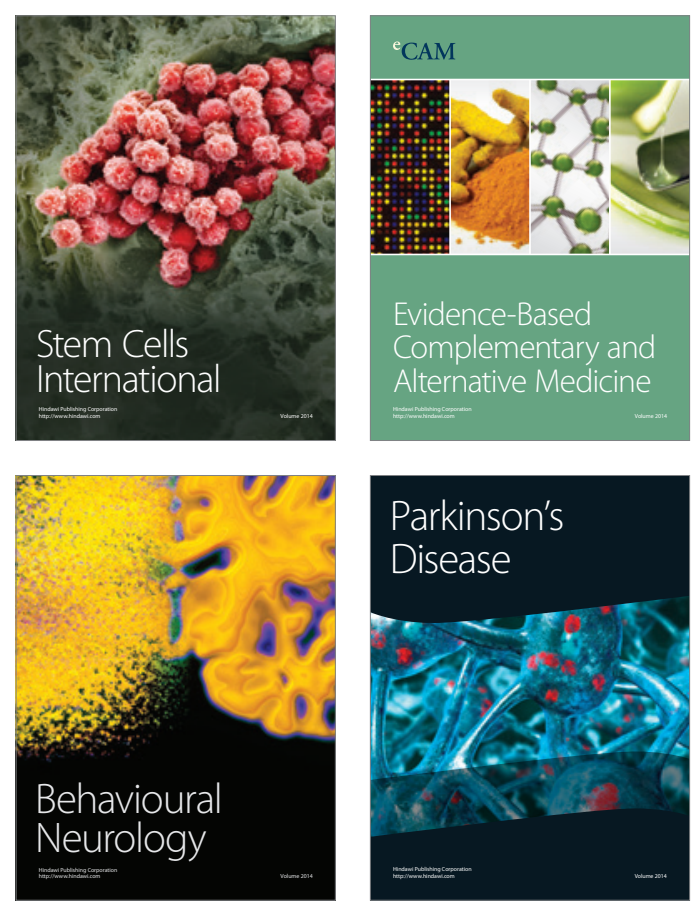

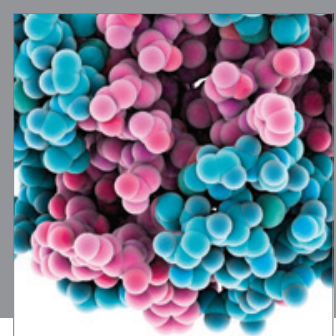

Journal of
Diabetes Research

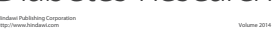

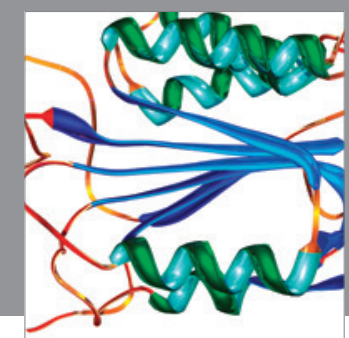

Disease Markers
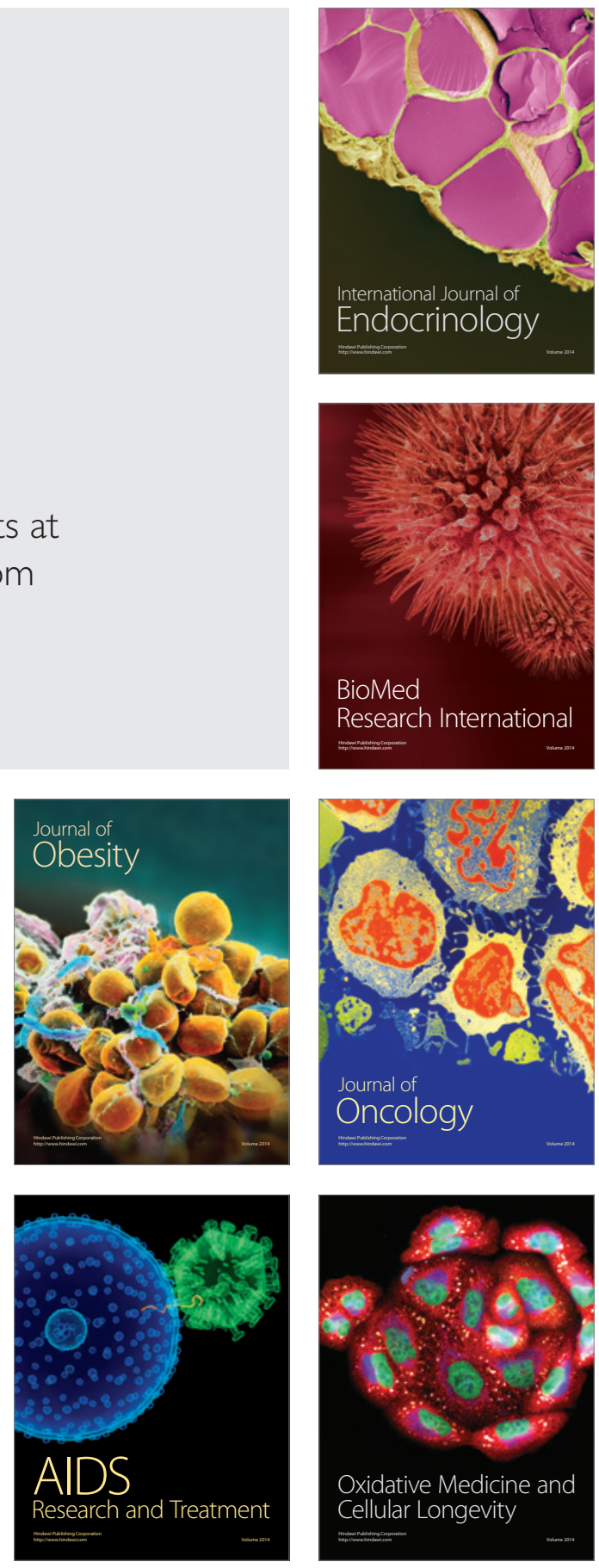